\title{
Need for Telehealth in the Era of Covid-19
}

\author{
Sara Ahmed Esmat Shoman', Tamer Emara², Heba Gamal Saber ${ }^{3}$ and Mohamed Farouk Allam ${ }^{1 *}$ \\ ${ }^{1}$ Department of Family Medicine, Faculty of Medicine, Ain Shams University, Egypt \\ ${ }^{2}$ Department of Neurology, Faculty of Medicine, Ain Shams University, Egypt
}

${ }^{3}$ Department of Geriatrics and Gerontology, Faculty of Medicine, Ain Shams University, Egypt

Corresponding author: Mohamed Farouk Allam, Department of Family Medicine, Faculty of Medicine, Ain Shams University, 11566

Abbasia, Cairo, Egypt

\begin{tabular}{|c|}
\hline ARTICLE INFO \\
\hline Received: 幽 October 13, 2020 \\
\hline Published: October 30, 2020 \\
\hline
\end{tabular}

Citation: S Ahmed Esmat Shoman, T Emara, H Gamal Saber, M Farouk Allam. Need for Telehealth in the Era of Covid-19. Biomed J Sci \& Tech Res 31(3)-2020. BJSTR. MS.ID.005118.

Keywords: Telehealth; Healthcare Services; Virtual care; COVID-19; Remote treatment; Pandemic

\begin{abstract}
Telehealth means the usage of telecommunications and virtual technology in facilitating healthcare services in far areas. Telehealth is a multi- phasic system as it serves patients and healthcare workers (HCWs) by providing them with helpful tool for proper healthcare services and giving HCWs the chance to receive training sessions and conducting research. Telehealth proved to be practical in outbreaks and emergency settings as it provides platforms that are easy in usage and accessible for health, education, training, and others. Since the beginning of COVID-19 pandemic, telehealth services have been of great importance in diagnosis, prevention, treatment, and control of corona virus infection. Telehealth application during COVID-19 pandemic appears in screening and triaging the patients according to their symptoms through phone screening, online tools, mobile applications, and virtual visits to evaluate their conditions, and making the appropriate decisions. Also, telehealth is used in contacts tracing to determine whether they are infectious and need to be quarantined. The importance of telehealth extends to include following up of home isolated patients with mild and moderate symptoms to avoid overcrowding of healthcare facilities, in addition to delivering care by a diverse experienced team to provide and adjust treatment plan. Finally, telehealth sustains continuous care by keeping away from negative outcomes that could occur by delaying due to COVID-19.
\end{abstract}

\section{Mini Review}

Telehealth is growing fast and got popular in both developed and developing countries, especially with the increasing struggle of communicable and non-communicable diseases in order to reach the highest impact on health outcomes [1]. Telehealth means the usage of telecommunications and virtual technology in facilitating healthcare services in far areas. Telehealth could help both patients and healthcare workers (HCWs) to deliver comprehensive easily accessible services, as a step toward universal health coverage (WHO, 2019). It helps in conveying health service to chronically ill and elderly while staying at home, as well as HCWs serving at isolated areas to receive consultations and guidance for diagnosis and ease referral system [2]. Application of telehealth is focused in four sectors:

A. real time telehealth used in consultation, diagnosis and treatment.
B. store-and-forward section used to send electronic medical records to a specialist to help in cases and to perform services rather than face-to-face approach.

C. remote patient monitoring and

D. mobile health as online services and mobile phone applications [3].

Telehealth application helps in many domains as providing continuous care in chronic diseases and deliver needed information for them; for example, giving opportunity to deliver educating messages on diabetes care, healthy lifestyle, mental health awareness, antenatal and perinatal care. In addition, it has special importance in low- and middle-income countries as they are in need for technology in health services to achieve powerful, accurate and fast health system in diagnosis, treatment and referring system [4]. 
Telehealth is a multi- phasic system as it serves patients and HCWs by providing them with helpful tool for proper healthcare services and giving HCWs the chance to receive training sessions and conducting research. Also, for undergraduate and postgraduate medical students, telehealth could give an important help for the education systems [5]. Telehealth benefits are increasing access to expertise in difficultly reached geographical areas with no available medical teams and may be used as the fast first aid, as well as it could minimize costs of hospitals, as patients can be monitored remotely even from home. As for barriers especially in developing countries are the unavailable infrastructure and the resistance of the patients and HCWs in adaption, together with the legal aspects [6]. Many countries globally started applying telehealth in providing health services in their primary healthcare settings in teleconsultation and distance education in both developing countries as India and Brazil $[7,8]$ and developed countries with well-established health systems as Australia and UK $[9,10]$; . On the contrary, the situation in the East Mediterranean region is still in need for a lot of work to be done as not many countries implemented telehealth in their health care settings, though steps are being taken toward applying it [11].

Telehealth proved to be practical in outbreaks and emergency settings as it provides platforms that are easy in usage and accessible for health, education, training and others. Telehealth facilitates screening patients and treating critically ill ones without face to face encountering [12]. Since the beginning of COVID-19 pandemic, telehealth services have been of great importance in diagnosis, prevention, treatment and control of corona virus infection. Till date, evidence has shown that the best strategy to prevent spread of COVID-19 infection is the social distancing and avoiding being exposed. Usage of telehealth in its different forms as live video conferencing and mobile calls allow medical professionals to provide medical consultation, patients triaging, home monitoring during recovery and regular checking of oxygen level and respiratory rate. It also allows the public to easily ask about the symptoms and modes of infection control, thus improve public awareness of the disease [13]. A recent systemic review on the role of telehealth during COVID-19 outbreak revealed a significant impact of telehealth in preventing morbidities and avoiding population who need health services from attending high risk areas as hospitals. Role of telehealth in management during COVID-19 showed high importance in avoiding direct contact. It minimizes the hazard of exposure to infection, especially in patients with chronic diseases (e.g. cancer, diabetes, chronic obstructive lung disease and systemic lupus erythematosus) requiring continuous check-up, as well as in elderly population as they can access health services remotely. As regards COVID-19 patients, telehealth is crucial in their management as it allows integration of several healthcare sectors for providing high level of care and reaching the best outcome for them. Also, it is of great benefit in ensuring healthcare providers safety and minimizing the burden of high flow and exposure on the health system [14].

Telehealth application during COVID-19 pandemic appears in screening and triaging the patients according to their symptoms through phone screening, online tools, mobile applications and virtual visits to evaluate their conditions, and making the appropriate decisions. Also, telehealth is used in contacts tracing to determine whether they are infectious and need to be quarantined. The importance of telehealth extends to include following up of home isolated patients with mild and moderate symptoms to avoid overcrowding of healthcare facilities, in addition to delivering care by a diverse experienced team to provide and adjust treatment plan. Finally, telehealth sustains continuous care by keeping away from negative outcomes that could occur by delaying due to COVID-19 [15]. COVID-19 pandemic has a massive psychological effect and impact on patients and their family members, healthcare providers and populations during lockdown. Telehealth with basic psychological health service could be very practical in providing support to the general population, patients, caregivers, and HCWs through an easy and appropriate way $[16,17]$. In conclusion, telehealth could be fundamental for improving healthcare services and in fighting COVID-19 outbreak all over the globe in all aspects.

\section{References}

1. Scott R, Mars M (2015) Telehealth in the developing world: current status and future prospects. Smart Homecare Technol Telehealth 3: 2537.

2. Wernhart A, Gahbauer S, Haluza D, Helve 0 (2019) eHealth and telemedicine: Practices and beliefs among healthcare professionals and medical students at a medical university. PLoS One 14(2): e0213067.

3. Marcoux RM, Vogenberg FR (2016) Telehealth: Applications from a Legal and Regulatory Perspective. P T 41(9): 567-570.

4. Ashford M, Rasekaba T, Wallwiener S, Aggarwal S (2018) eHealth as the Next-Generation Perinatal Care: An Overview of the Literature. J Med Internet Res 20(6): e202.

5. Rutledge CM, KOtt K, Schweickert PA, Poston R, Fowler C, et al. (2017) Telehealth and eHealth in nurse practitioner training: current perspectives. Adv Med Educ Pract 8: 399-409.

6. De Souza CHA, Morbech RA, Steinman M, Hors CP, Bracco MM, et al. (2017) Barriers and Benefits in Telemedicine Arising Between a HighTechnology Hospital Service Provider and Remote Public Healthcare Units: A Qualitative Study in Brazil. Telemed J E Health 23(6): 527-532.

7. Praveen KBA, Ali SS (2013) Telemedicine in Primary Health Care: The Road Ahead. Int J Prev Med 4(3): 377-378.

8. Deniz PR, Ribeiro SFJ, De Araujo Novaes MM (2016) Providing Telehealth Services to a Public Primary Care Network: The Experience of RedeNUTES in Pernambuco, Brazil. Telemed J E Health 22(8): 694.

9. Raven M, Butler C, Bywood C (2013) Video-based telehealth in Australian primary health care: current use and future potential. Aust J Prime Health 19(4): 283-286.

10. Nnamoko N, Arshad F, Hammond L, Mcpartland S, Patterson P (2016) Telehealth in Primary Health Care: Analysis of Liverpool NHS Experience, in Dhiya Al-Jumeily; Abir Hussain; Conor Mallucci \& Carol Oliver, ed., Applied Computing in Medicine and Health, Elsevier and Morgan Kaufmann, Amsterdam 269-286. 
11. Alsadan M, El Metwally A, Ali A, Jamal A, Khalifa M et al. (2015) Health Information Technology (HIT) in Arab Countries: A Systematic Review Study on HIT Progress. J Health Inform Dev Ctries 9(2): 32-49.

12. Anthony Jnr B (2020) Use of Telemedicine and Virtual Care for Remote Treatment in Response to COVID-19 Pandemic. J Med Syst 44(7): 132.

13. Gao Y, Liu R, Zhou Q, Wang X, Huang L, et al. (2020) Application of telemedicine during the coronavirus disease epidemics: a rapid review and meta-analysis. Anns Transl Med 8(10): 626.

14. Monaghesh E, Hajizadeh A (2020) The role of telehealth during COVID-19 outbreak: a systemic review bases on current evidence. BMC Public Health 20: 1193.

\section{ISSN: 2574-1241}

DOI: 10.26717/BJSTR.2020.31.005118

Mohamed Farouk Allam. Biomed J Sci \& Tech Res

(C) This work is licensed under Creative

Submission Link: https://biomedres.us/submit-manuscript.php
15. CDC (2020), National Center for Immunization and Respiratory Diseases (NCIRD), Division of Viral Diseases. Uses of Telehealth during COVID-19 in Low Resource on-US Settings, USA.

16. Zhou X, Snoswell CL, Harding LE, Bambling M, Edirippulige S, et al (2020) The role of telehealth in reducing the mental health burden from COVID-19. Telemed e-Health 26(4): 377.

17. World health Organization [WHO] (2020). eHealth at WHO, USA.

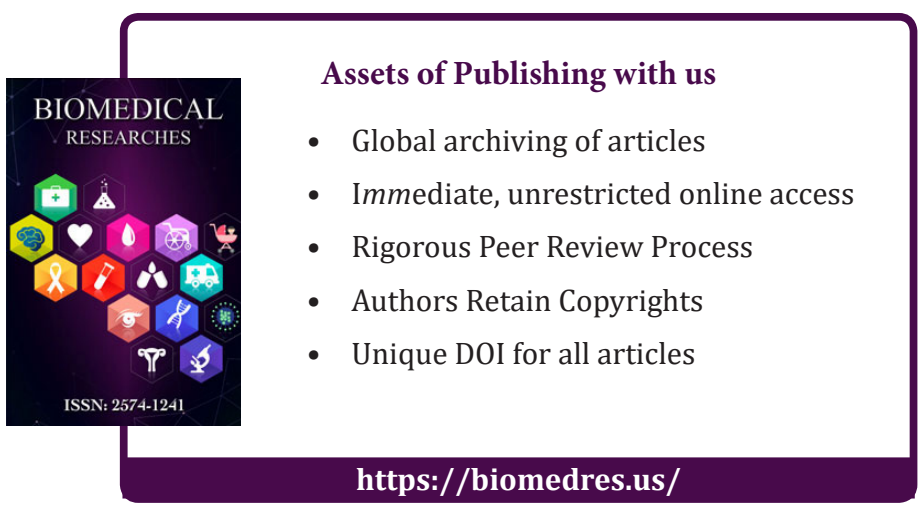

